\title{
Contratransferência no atendimento inicial de vítimas de violência sexual e urbana: uma pesquisa qualitativa/quantitativa
}

\author{
Countertransference in the initial care of victims of sexual and urban violence: a \\ qualitative-quantitative research
}

\author{
Mariana Eizirik $^{1}$, Guilherme Polanczyk ${ }^{2}$, Sidnei Schestatsky ${ }^{3}$, Maria Amélia Jaeger ${ }^{4}$, Lúcia Helena \\ Freitas Ceitlin ${ }^{5}$
}

${ }^{1}$ Médica psiquiatra. Mestranda em Psiquiatria, Universidade Federal do Rio Grande do Sul, (UFRGS), Porto Alegre, RS. ${ }^{2}$ Médico psiquiatra. Mestre em Psiquiatria, UFRGS. ${ }^{3}$ Professor adjunto, Departamento de Psiquiatria e Medicina Legal, UFRGS. Coordenador, Núcleo de Estudos e Tratamento do Trauma Psíquico (NET-TRAUMA), Hospital de Clínicas de Porto Alegre (HCPA), Porto Alegre, RS. ${ }^{4}$ Psicóloga. Mestre em Psicologia, Pontifícia Universidade Católica do Rio Grande do Sul (PUCRS), Porto Alegre, RS. ${ }^{5}$ Professora adjunta, Departamento de Psiquiatria e Medicina Legal, UFRGS. Coordenadora, NET-TRAUMA, HCPA.

Estudo realizado no Departamento de Psiquiatria e Medicina Legal, UFRGS, e no NET-TRAUMA, HCPA.

\section{Resumo}

Objetivo: Avaliar a contratransferência dos terapeutas durante o atendimento inicial de pacientes mulheres vítimas de violência sexual e urbana, investigando a influência do gênero do terapeuta e da natureza e momento do trauma.

Método: A amostra foi composta por 36 relatos redigidos por médicos residentes de psiquiatria do Hospital de Clínicas de Porto Alegre, oriundos do atendimento de 36 pacientes. Este estudo utilizou métodos qualitativos e quantitativos para a análise dos seus dados. Os relatos foram classificados em seis grupos, conforme o gênero do terapeuta e a natureza do trauma. Foi realizada a análise de conteúdo dos relatos. Associou-se uma análise estatística dos dados.

Resultados: Houve predomínio de sentimentos de aproximação nos terapeutas de ambos os sexos no atendimento de vítimas de violência sexual. Entre terapeutas mulheres, a natureza do trauma (sexual ou urbano) não influenciou os padrões contratransferenciais $(\mathrm{p}=0,7)$. Entre os terapeutas homens, ao contrário, a natureza do trauma influenciou de forma significativa $(\mathrm{p}=0,044)$ o padrão contratransferencial, havendo um número elevado de sentimentos de distanciamento nos relatos de atendimentos de vítimas de violência urbana.

Conclusões: Houve um predomínio de sentimentos de aproximação dos terapeutas de ambos os sexos no atendimento inicial de pacientes vítimas de violência sexual. Foi observado um predomínio de sentimentos de distanciamento nos terapeutas homens que atenderam vítimas de violência urbana. Mais estudos são necessários para uma melhor compreensão das relações terapêuticas nos atendimentos de vítimas de trauma psíquico.

Descritores: Contratransferência, violência sexual, violência urbana, trauma psíquico, psicoterapia.

\begin{abstract}
Objective: To assess therapists' countertransference during initial care of female victims of sexual and urban violence with the aim of investigating influence of therapist's gender, type and moment of trauma.

Methods: The sample comprised 36 reports written by psychiatry residents at Hospital de Clínicas de Porto Alegre based on the care provided to 36 patients. This study used qualitative and quantitative methods of data analysis. Reports were classified into six groups according to therapist's gender and type of trauma. A content analysis and a statistical analysis of the data were performed.
\end{abstract}


Results: Therapists of both genders presented a prevalence of feelings of closeness when providing care to victims of sexual violence. Among female therapists, type of trauma (sexual or urban) did not have an influence on countertransference patterns ( $p=$ $0,7)$. On the other hand, among male therapists, type of trauma had a significant influence $(p=0,044)$ on countertransference, with a high rate of feelings of distance in reports of care provided to victims of urban violence.

Conclusions: Therapists of both genders presented a prevalence of feelings of closeness when providing initial care to patients victims of sexual violence. Male therapists who provided care to victims of urban violence presented a prevalence of feelings of distance. Further studies are needed for a better understanding of therapeutic relationships in the care of victims of psychic trauma. Keywords: Countertransference, sexual violence, urban violence, psychic trauma, psychotherapy.

\section{Introdução}

O conceito de contratransferência, introduzido por Freud $^{1}$ e ampliado por outros autores ${ }^{2-4}$, é central na teoria e na técnica psicanalítica atuais, que entendem os sentimentos despertados pelo paciente no terapeuta como ferramentas poderosas para a compreensão da comunicação e do mundo interno do paciente. A partir do reconhecimento crescente da importância da mente do terapeuta e do campo terapêutico estabelecido entre a dupla, novas teorias e pesquisas empíricas vêm sendo desenvolvidas ${ }^{5-7}$. Atualmente, a atenção sobre a contratransferência é ressaltada já no ensino médico, sugerindo-se a mesma como um instrumento importante no desenvolvimento de uma adequada relação médico/ paciente - incluindo casos de tratamento basicamente farmacológico.

Gabbard $^{8}$ ressalta haver, atualmente, consenso em considerar a utilidade da contratransferência no entendimento do paciente, sendo esta uma "criação conjunta" da dupla terapêutica. Mais ainda, tem-se discutido amplamente as características da pessoa real do terapeuta, como gênero, etapa de treinamento e faixa etária, que estariam associadas a padrões contratransferenciais constantes e que teriam uma implicação direta na escolha de terapeutas conforme características da situação clínica e do paciente ${ }^{9-11}$.

Estudos empíricos sobre contratransferência ainda não são comumente encontrados na literatura, embora sua importância fique clara no recente artigo de Betan et al. ${ }^{12}$ Através da análise fatorial de uma escala para avaliação de processos contratransferenciais, foram encontradas oito classes de reações distintas, independentes da orientação teórica do terapeuta, associadas com diferentes tipos de patologia de eixo II dos pacientes. Apesar da singularidade de cada dupla terapêutica, foram evidenciadas correlações significativas entre sentimentos contratransferenciais específicos e sintomas de transtornos de personalidade. Isso sugere que as repostas contratransferenciais podem surgir em padrões coerentes e previsíveis, fortalecendo seu uso na compreensão diagnóstica e no entendimento e tratamento dos pacientes. Os autores ressaltam a possibilidade e a relevância de mapear padrões contratransferenciais despertados por pacientes com diferentes diagnósticos psiquiátricos ou naqueles que compartilham certas experiências, como história de violência sexual ${ }^{12}$.

O estudo dos sentimentos despertados por pacientes vítimas de violência sexual mostra-se importante, já que, além de intensos, muitas vezes esses sentimentos constituem-se como barreiras para o sucesso do tratamento ${ }^{13}$. Observa-se um crescente número de estudos relacionados à violência e à abordagem terapêutica oferecida às suas vítimas, dentre os quais se encontra ainda pouco referente a contratransferência.

Os terapeutas podem ser os primeiros a identificar, através de si mesmos, a raiva, o medo, a vergonha e a insegurança dos pacientes, já que estes, inicialmente, não costumam estar conscientes de suas experiências afetivas. São sentimentos muitas vezes intoleráveis e contraditórios, habitualmente reproduzidos e vividamente reexperimentados durante o tratamento pela dupla terapêutica, podendo atemorizar a ambos e criar impasses se não forem adequadamente reconhecidos e elaborados ${ }^{14}$. Vítimas de trauma estão super-representadas em situações de impasses ou de fracassos terapêuticos, tendo menores taxas de sucesso no tratamento quando comparadas a pessoas sem história de trauma ${ }^{15}$. Sabe-se que a probabilidade de ocorrência de impasse ao longo de um tratamento psicoterápico está relacionada às "condições de ordem inconsciente que regem a dupla terapêutica"16, incluindo-se aqui a não-compreensão dos processos transferenciais/contratransferenciais envolvidos ${ }^{15}$.

O momento da vida do paciente em que a situação de violência sexual ocorreu associa-se ao desenvolvimento de formas específicas de psicopatologia, principalmente transtornos de humor e transtornos de personalidade, o que poderia estar relacionado também a respostas contratransferenciais específicas ${ }^{17-21}$.

A violência é a segunda principal causa de mortalidade global do Brasil, que é o quarto país com maior prevalência de homicídios no mundo. A taxa de mortalidade por causas externas é de $14,58 \%$, sendo a taxa por 100.000 habitantes de homicídios dolosos 22, a de roubos 511 e a de estupro $16^{22}$. Estudos de prevalência mostram taxas de abuso sexual ao longo da vida variando entre 15 e $25 \%$ na população feminina ${ }^{23}$. Essa situação 
alarmante cria uma grande demanda para o serviço de saúde do país, principalmente para profissionais da área da saúde mental, que se vêem frente às vítimas diretas dessas situações e aos seus familiares, reforçando a necessidade de estudos que enriqueçam a compreensão do tema. A possibilidade de entender as reações emocionais despertadas nesses contatos pode facilitar aos terapeutas o reconhecimento e manejo dos seus sentimentos contratransferenciais. Assim, haveria menores chances destes se tornarem obstáculos maiores para o desenvolvimento e manutenção de uma relação terapêutica adequada.

Considerando que o atendimento de vítimas de violência, especialmente de natureza sexual, constituise em um desafio aos profissionais da saúde mental sejam eles psiquiatras clínicos, psicoterapeutas ou psicanalistas - e que o número de pessoas expostas a essas situações vem aumentando dramaticamente, este estudo buscou avaliar as respostas contratransferenciais despertadas nos terapeutas durante o atendimento inicial de pacientes vítimas de traumas psíquicos. Foi investigada a influência do gênero do terapeuta e da natureza e momento do trauma nessas reações. Tendo em vista a complexidade do fenômeno em estudo (contratransferência), optou-se pela combinação dos métodos de análise qualitativos e quantitativos, no sentido de ampliar a fidedignidade dos achados.

\section{Método}

\section{Amostra}

A amostra foi composta por 36 relatos redigidos por médicos residentes do segundo ano de psiquiatria do Hospital de Clínicas de Porto Alegre (HCPA), oriundos do atendimento de 36 pacientes do sexo feminino atendidas no Núcleo de Estudos e Tratamento do Trauma Psíquico (NET-TRAUMA) do HCPA, durante 9 meses consecutivos, nos anos de 2005 a 2006. Esse ambulatório oferece atendimento aos pacientes vítimas de trauma psíquico encaminhados por serviços de atenção primária, secundária e terciária da cidade. Após a primeira consulta com a paciente, o terapeuta era solicitado a registrar de forma livre e anônima o que havia sentido durante o atendimento.

Os critérios de inclusão das pacientes foram: 1) sexo feminino; 2) vítimas de violência sexual até os 12 anos de idade (na infância) ou nos últimos 3 meses (atual); ou 3) vítimas de assaltos ou que tiveram familiar morto vitimado por violência urbana (outros traumas). Critérios de exclusão eram: 1) incapacidade de relatar a história de forma adequada; e 2) presença de sintomatologia intensa que justificasse a indicação de internação psiquiátrica.
Restringiu-se a amostra deste estudo à população feminina pela pouca procura de pacientes homens por atendimento no NET-TRAUMA.

\section{Análise de conteúdo}

Os relatos foram classificados em seis grupos conforme o gênero do terapeuta e a natureza do trauma (violência sexual na infância, violência sexual atual e outros traumas) por um dos autores do estudo. Nesse momento, foram excluídas dos relatos todas as menções feitas ao momento e à natureza do trauma, assim como ao gênero do terapeuta, sem prejuízo ao conteúdo dos mesmos. Posteriormente, outros três autores realizaram a análise de conteúdo dos relatos, de forma cega, sem conhecimento de suas classificações.

A análise de conteúdo ${ }^{24}$ é uma metodologia de pesquisa utilizada para descrever e interpretar o conteúdo de documentos e textos, ajudando a reinterpretar as mensagens e alcançar uma compreensão de seus significados em um nível que vai além de uma leitura comum ${ }^{25}$. É constituída de cinco etapas: 1) preparação das informações (em que se seleciona o material a ser utilizado na pesquisa e se inicia o processo de codificação); 2) unitarização (quando são definidas as unidades de análise); 3) categorização ou classificação das unidades em categorias previamente definidas pelos autores (quando os dados são agrupados considerando-se a parte comum existente entre eles, em um processo de redução e síntese); 4) descrição; e 5) interpretação (etapa em que se discute os resultados, aprofundando-se a compreensão do material obtido).

Iniciou-se a análise de conteúdo com a leitura do material, seguindo-se a divisão em unidades de análise, definidas como palavras representativas dentro de uma frase, como o exemplo: "inicialmente, a paciente me despertou desesperança importante e muita pena, bastante desconforto também", em que foram retiradas as palavras "desesperança", "pena" e "desconforto" como unidades de análise dessa frase. Foi possível estabelecer duas categorias de interesse a partir dos sentimentos relatados, agrupando-se as unidades de análise em sentimentos de aproximação ou de distanciamento. As unidades não caracterizadas por tais sentimentos foram desconsideradas.

Os processos de unitarização e categorização foram realizados individualmente por dois autores e, em um segundo momento, em conjunto, através de consenso. $\mathrm{Na}$ falta de consenso, a decisão foi tomada por um autor sênior, após discussão do material.

\section{Análise estatística}

As características demográficas das pacientes, estratificadas pela natureza do trauma psíquico sofrido, 
foram comparadas através da ANOVA para variáveis contínuas (idade) e Teste Exato de Fisher para variáveis categóricas (etnia, estado civil, escolaridade). Foi utilizado o Teste Exato de Fisher para comparação das categorias de sentimentos entre gênero e natureza do trauma, seguido por um teste de comparações múltiplas, utilizando o programa WINPEPI, Módulo Compare2, versão 1.45. Foi considerado como estatisticamente significativo um valor de $\mathrm{p} \leq 0,05$.

\section{Questões éticas}

Pacientes e terapeutas não foram identificados em nenhum momento do estudo. Todos os participantes assinaram um termo de consentimento informado e esclarecido. Este trabalho foi aprovado pelo Comitê de Ética em Pesquisa do HCPA.

\section{Resultados}

Durante o período de estudo, 36 pacientes preencheram os critérios de inclusão. Destas, 13 eram vítimas de violência sexual na infância; 15 , de violência sexual atual; e 8 , de outros traumas. Todos os relatos dos sentimentos contratransferenciais descritos após as primeiras consultas de todas as pacientes foram incluídos neste estudo. A Tabela 1 apresenta as características demográficas das pacientes, não tendo sido encontradas diferenças estatisticamente significativas entre os grupos. $\mathrm{O}$ número total de unidades de análise destes relatos foi de 141 , sendo que 98 corresponderam a sentimentos de aproximação, e 43, a sentimentos de distanciamento.

Tabela 1 - Características demográficas das pacientes incluídas no estudo, de acordo com a natureza do trauma

\begin{tabular}{lcccc}
\hline & $\begin{array}{c}\text { Violência sexual na } \\
\text { infância }(\mathbf{n}=\mathbf{1 3})\end{array}$ & $\begin{array}{c}\text { Violência sexual } \\
\text { atual }(\mathbf{n}=\mathbf{1 5})\end{array}$ & $\begin{array}{c}\text { Outros traumas } \\
(\mathbf{n}=\mathbf{8})\end{array}$ & \\
\hline Idade, média (DP) & $40,15(14,5)$ & $29,71(14,82)$ & $37,62(15,38)$ & $\mathrm{p}=0,17^{*}$ \\
Etnia, n (\%) & & & & \\
$\quad$ Branca & $12(93)$ & $13(86,6)$ & $7(87,5)$ & \\
$\quad 1(7)$ & $2(13,3)$ & $1(12,5)$ & $\mathrm{p}=0,99^{\dagger}$ \\
$\quad$ Negra & & & & \\
Estado civil, n (\%) & $7(53,8)$ & $4(26,7)$ & $5(62,5)$ & \\
$\quad$ Casada & $6(46,2)$ & $11(73,3)$ & $3(37,5)$ & $\mathrm{p}=0,21^{\dagger}$ \\
$\quad$ Solteira/divorciada/viúva & & & & \\
Escolaridade, n (\%) & $5(38,5)$ & $7(46,7)$ & $4(50)$ & \\
$\quad$ Ensino fundamental & $8(61,5)$ & $8(53,3)$ & $4(50)$ & $\mathrm{p}=0,83^{\dagger}$ \\
$\quad$ Ensino médio/superior & & & &
\end{tabular}

$\mathrm{DP}=$ desvio padrão; $\mathrm{n}=$ freqüência absoluta; $\%=$ freqüência relativa

*ANOVA.

${ }^{\dagger}$ Teste exato de Fisher.

Foi investigada, em separado, a influência da natureza do trauma psíquico sobre o padrão contratransferencial de terapeutas do sexo masculino e feminino. Entre terapeutas do sexo feminino, a natureza do trauma não influenciou os padrões contratransferenciais encontrados $(p=0,7)$ (Tabela 2). As seguintes vinhetas de atendimentos de pacientes com os três diferentes traumas, realizados por terapeutas mulheres, ilustram os relatos. "Senti apreensão por perceber que isso pode acontecer com qualquer pessoa, com desejo de que não tivesse ocorrido com a paciente"; "Senti confusão no início da sessão e, após, sensação de estar mexendo em um problema muito grande, de impotência e, ao mesmo tempo, satisfação por saber que conseguiria ajudar um pouco no meio de tanta confusão"; "Senti angústia, pena da paciente e raiva em relação ao responsável pelo trauma"; "Senti que a paciente se comportava como uma criança, tive vontade de cuidála e de, ao mesmo tempo, fazer com que ela voltasse a funcionar como antes do trauma. Também me despertou curiosidade e interesse"; "Inicialmente me despertou desesperança importante e muita pena, além de bastante desconforto. Ao longo da consulta, senti tristeza e vontade de ajudar, porém com uma grande sensação de imobilidade".

Entre os terapeutas homens, a natureza do trauma influenciou de forma significativa $(\mathrm{p}=0,044)$ o padrão contratransferencial. Houve um número elevado de sentimentos de distanciamento nos relatos de 
atendimentos de pacientes vítimas de assalto ou com familiares mortos (55\%). Quando comparada aos outros grupos (violência sexual atual e violência sexual na infância), tal freqüência apresentou diferença estatisticamente significativa em relação à freqüência de sentimentos de distanciamento nos atendimentos de vítimas de violência sexual na infância ( 55 versus $14,3 \%$; $\mathrm{p}=0,025)$, não apresentando diferença significativa em comparação ao grupo de vítimas de violência sexual nos últimos 3 meses (55 versus $25 \% ; \mathrm{p}=0,18$ ). (Tabela 3 )

Tabela 2 - Freqüência das unidades de análise nos relatos de terapeutas mulheres de acordo com o tipo de trauma sofrido pelas pacientes atendidas

\begin{tabular}{lccc}
\hline & \multicolumn{2}{c}{ Unidades de análise, n (\%) } \\
\cline { 2 - 4 } & $\begin{array}{c}\text { Sentimentos de } \\
\text { aproximação }\end{array}$ & $\begin{array}{c}\text { Sentimentos de } \\
\text { distanciamento }\end{array}$ & \\
\hline Violência sexual atual & $32(74,4)$ & $11(25,6)$ & \\
Violência sexual na infância & $27(67,5)$ & $13(32,5)$ & $\mathrm{p}=0,7^{*}$ \\
Outros traumas & $9(75)$ & $3(25)$ & \\
\hline
\end{tabular}

$\mathrm{n}=$ freqüência absoluta de unidade de análises; \% = freqüência relativa.

* Teste exato de Fisher.

Tabela 3 - Freqüência das unidades de análise nos relatos de terapeutas homens de acordo com a natureza do trauma sofrido pelas pacientes atendidas

\begin{tabular}{lccc}
\hline & \multicolumn{2}{c}{ Unidades de análise, n (\%) } \\
\cline { 2 - 4 } & $\begin{array}{c}\text { Sentimentos de } \\
\text { aproximação }\end{array}$ & $\begin{array}{c}\text { Sentimentos de } \\
\text { distanciamento }\end{array}$ & \\
\hline Violência sexual atual & $9(75)$ & $3(25)^{*^{\dagger}}$ & \\
Violência sexual na infância & $12(85,7)$ & $2(14,3)^{* \S}$ & $\mathrm{p}=0,04^{\ddagger}$ \\
Outros traumas & $9(45)$ & $11(55)^{\dagger, \S}$ & \\
\hline
\end{tabular}

$\mathrm{n}=$ freqüência absoluta de unidade de análises; \% = freqüência relativa.

\$ Teste exato de Fisher.

Teste de comparações múltiplas: ${ }^{*}=\mathrm{p}>0,05 ;{ }^{\dagger}=\mathrm{p}>0,05 ;{ }^{\S}=\mathrm{p}=0,02$.

Seguem-se vinhetas de atendimentos realizados por terapeutas homens a vítimas de assalto ou que tiveram familiares assassinados: "Senti impotência no início da sessão, que foi gradativamente aumentando, sendo que o fim da sessão foi um alívio para mim, como se pudesse voltar a viver sem saber que eventos tão trágicos são possíveis com qualquer um de nós. Senti um predomínio de impotência, com desesperança e um grande desespero"; "Senti imobilidade e sensação de choque com os detalhes do trauma, por vezes com sentimento de medo e preocupação pela vulnerabilidade das pessoas às tragédias"; "De início, a paciente causou certa antipatia, certo distanciamento, talvez com dificuldade em me identificar com a situação e com a tendência a desvalorizar o sofrimento da paciente".

Pelo que pode ser observado a partir das verbalizações acima, o impacto desses atendimentos acionou nos terapeutas homens sentimentos de impotência, desesperança e medo de estar sendo envolvido em uma situação além de sua capacidade de manejo, o que possivelmente despertou ansiedades mais profundas, de natureza confusional ou persecutória. Estas podem ter levado a atitudes de afastamento e estranheza para com as pacientes, rechaçando o perigo de maiores identificações.

Foi analisada a influência do gênero do terapeuta sobre os sentimentos contratransferenciais, estratificada pela natureza do trauma. Evidenciou-se que terapeutas do sexo masculino e feminino não apresentaram padrão de resposta contratransferencial significativamente diferente no atendimento de vítimas de violência sexual na infância $(\mathrm{p}=0,30)$ ou nos últimos 3 meses $(\mathrm{p}=$ $0,99)$ e de vítimas de assalto ou pacientes que tiveram um familiar morto $(\mathrm{p}=0,14)$ (Tabela 4$)$. 
Tabela 4 - Comparação das freqüências das unidades de análise de acordo com o gênero do terapeuta, estratificada pela natureza do trauma vivenciado pelas pacientes

\begin{tabular}{lcccc}
\hline Trauma psíquico & $\begin{array}{c}\text { Gênero do } \\
\text { terapeuta }\end{array}$ & \multicolumn{2}{c}{ Unidades de análise - n (\%) } & \\
\cline { 3 - 5 } & & $\begin{array}{c}\text { Sentimentos de } \\
\text { aproximação }\end{array}$ & $\begin{array}{c}\text { Sentimentos de } \\
\text { distanciamento }\end{array}$ & Valor de p \\
\hline Violência sexual atual & Feminino & $32(74,4)$ & $11(25,6)$ & 0,99 \\
& Masculino & $9(75)$ & $3(25)$ & \\
Violência sexual na infância & Feminino & $27(67,5)$ & $13(32,5)$ & 0,3 \\
& Masculino & $12(85,7)$ & $2(14,3)$ & \\
Outros traumas & Feminino & $9(75)$ & $3(25)$ & 0,14 \\
& masculino & $9(45)$ & $11(55)$ & \\
\hline
\end{tabular}

$\mathrm{n}=$ freqüência absoluta de unidades de análise; \% = freqüência relativa.

\section{Discussão}

Este estudo utilizou um método qualitativo, a análise de conteúdo, para avaliar as repostas contratransferenciais de terapeutas em formação no atendimento inicial de pacientes do sexo feminino vítimas de violência sexual e urbana. Os resultados mostram predomínio de sentimentos de aproximação quando comparados aos sentimentos de distanciamento nos relatos dos terapeutas. $\mathrm{O}$ atendimento de vítimas de violência urbana provocou, em terapeutas homens, um maior número de sentimentos de distanciamento do que o atendimento a vítimas de violência sexual na infância ( 55 versus $14,3 \%$; $\mathrm{p}=0,04)$.

A exposição à violência sexual na infância está associada ao desenvolvimento de psicopatologia na idade adulta ${ }^{17-19,26,27}$, que, de acordo com achados empíricos, evocaria respostas contratransferenciais específicas. Betan ${ }^{12}$ encontrou associações significativas entre classes distintas de contratransferência e sintomas específicos de transtornos de personalidade, assim como Brody ${ }^{21}$ e $\mathrm{Mclntyre}^{20}$, que evidenciaram uma relação igualmente significativa entre o diagnóstico do paciente e o padrão contratransferencial. Em oposição a esses achados, Holmqvist $^{28}$ não evidenciou associações entre a organização de personalidade do paciente e os tipos de sentimentos do terapeuta, contribuindo para a discussão desse tema, ainda controverso.

Os resultados do presente estudo mostraram um predomínio de respostas contratransferenciais de aproximação, como interesse, empatia, tristeza, tanto em pacientes vítimas de violência sexual na infância como nos últimos 3 meses. Esse achado pode estar relacionado à ocorrência de psicopatologia específica após violência sexual em mulheres adultas sem história de violência sexual na infância, conforme demonstrado por Faravelli ${ }^{29}$. Essa associação excluiria a especificidade do momento do trauma no desenvolvimento de sintomas e transtornos e, conseqüentemente, na produção de reações características nos terapeutas.

O estudo de pacientes vítimas de traumas nãosexuais deveu-se à hipótese de que haveria uma psicopatologia característica em pacientes vítimas de violência sexual, conforme o achado de Faravelli ${ }^{29}$, com conseqüentes diferenças nos sentimentos contratransferenciais. Nossos achados revelaram um predomínio de sentimentos de aproximação em relação a todos os grupos de pacientes, independentemente da natureza do trauma e do gênero do terapeuta, com exceção das pacientes vítimas de trauma não-sexual atendidas por homens.

Há um número reduzido de estudos acerca da contratransferência no atendimento de pacientes vítimas de traumas não-sexuais ${ }^{30,31}$, além da falta de dados que relacionem esse tema ao gênero do terapeuta. Uma hipótese a ser avaliada é a de que a predominância de sentimentos de distanciamento vivenciados pelos terapeutas homens no atendimento de vítimas de violência urbana, não-sexual, esteja relacionada a uma sensação de vulnerabilidade, considerando a maior prevalência de crimes violentos contra homens no Brasil, o que não ocorreria em relação à violência sexual.

A influência do gênero do terapeuta no desenvolvimento da relação com o paciente e no tratamento vem sendo investigada com interesse crescente. Uma idéia comum entre muitos autores psicanalíticos é a de que não é o gênero "real" do terapeuta que irá influenciar de forma mais relevante o processo de tratamento, e sim suas capacidades técnicas, experiência profissional, aspectos de personalidade e autoconhecimento ${ }^{10,32,33}$. A relação da 
contratransferência com o gênero do terapeuta é tema central dessas questões, visto sua importância na prática psicanalítica e psicoterapêutica. Estudos empíricos com pacientes portadores de diagnósticos específicos mostram resultados divergentes. Zlotnick ${ }^{34}$ e Mclntyre $^{20}$ não encontraram diferenças nos sentimentos contratransferenciais em função do gênero do terapeuta no atendimento de pacientes com transtorno depressivo maior e transtorno de personalidade borderline. Já Latts $^{35}$, em um estudo com pacientes vítimas de violência sexual, observou um predomínio significativamente maior de respostas de evitação nos terapeutas homens em comparação às terapeutas mulheres.

Um estudo realizado em nosso meio avaliou o estado mental e as respostas contratransferenciais de psicoterapeutas homens e mulheres após a leitura de vinhetas clínicas reais, sendo uma referente a um caso de estupro, e outra, a um caso de luto. Foram identificadas reações contratransferenciais mais negativas após a leitura da vinheta do caso de estupro, sendo que as terapeutas mulheres mostraram-se mais reflexivas, e os terapeutas homens expressaram mais reações negativas, em ambos os $\operatorname{casos}^{36}$.

Os resultados do presente trabalho apontaram para uma similaridade das respostas contratransferenciais entre os gêneros do terapeuta no atendimento de pacientes vítimas de violência sexual. Os achados de Latts $^{35}$, de que as terapeutas mulheres conseguiriam utilizar mais a empatia do que os terapeutas homens no atendimento dessas pacientes, mesmo em contato com uma situação que lhes exporia de forma mais intensa ao sentimento de vulnerabilidade, não foram replicados na presente amostra.

É importante considerarmos que o relato feito pelo terapeuta ocorreu após o primeiro contato deste com a paciente e sua história, geralmente um momento de grande carga emocional para a dupla. Também é relevante o fato de serem todos terapeutas no início de sua formação profissional, com menos ferramentas para lidar com uma situação contratransferencial potencialmente difícil. Mesmo com essas características, que poderiam levar ao predomínio de respostas de distanciamento ou imobilidade, observou-se um maior número de relatos de sentimentos como curiosidade, desejo de ajudar, pena e interesse, em terapeutas de ambos os sexos. Pode-se supor que os terapeutas homens, assim como as terapeutas mulheres, conseguem utilizar a empatia de forma a moderar a ansiedade despertada na contratransferência durante o atendimento de mulheres vítimas de violência sexual.

É necessário entendermos os resultados no contexto das limitações do estudo. Trata-se de uma amostra clínica, com número reduzido de pacientes, atendidas por terapeutas em formação, em um programa dedicado ao tratamento de pacientes vítimas de violência. Essas características limitam a generalização dos resultados.

Em relação à etapa da formação profissional do terapeuta, há evidências de que esta se encontra associada à forma com que as reações contratransferenciais são manejadas ${ }^{20}$. Em nosso estudo, todos os terapeutas apresentavam-se no mesmo momento da formação profissional, o que torna essa variável estável em todos os grupos. Quanto ao número de pacientes estudadas, ainda que reduzido, é preciso considerar que há barreiras importantes para que vítimas de violência sexual busquem tratamento, o que dificulta o estudo dessa situação clínica.

Como se trata de uma situação de inegável impacto emocional, cabe, também, destacar que o uso do instrumental teórico psicanalítico apresenta aqui particular relevância. Não somente pelo seu uso em um projeto de pesquisa, mas também pela possibilidade dos sentimentos contratransferenciais despertados serem discutidos em supervisão e utilizados como bússola para orientar o manejo terapêutico subseqüente.

Utilizamos uma metodologia qualitativa, a análise de conteúdo, para que os relatos de sentimentos contratransferenciais - subjetivos - fossem entendidos com maior profundidade. Os instrumentos objetivos, embora relevantes, não são suficientes para captar a complexidade das experiências contratransferenciais, que acabam por ser reduzidas.

Este trabalho propõe-se a expor e discutir seus achados, levantando questões de pesquisa, sem o objetivo de comprovar os resultados obtidos, nem de generalizálos a outras populações. A compreensão adequada e profunda dos sentimentos contratransferenciais no atendimento de vítimas de violência pode trazer benefícios significativos à formação dos terapeutas, enfatizando a presença de conceitos básicos da psicanálise, assim como ao tratamento e ao prognóstico dos pacientes. Considerando a escassez de estudos sobre esse tema na população brasileira, sugerimos que investigações continuadas sejam realizadas, valendo-se de métodos qualitativos, quantitativos ou da união de ambos os enfoques, para seguir-se aprimorando o conhecimento nessa área de fenômenos tão complexos.

\section{Conclusões}

Os resultados deste estudo mostram um predomínio de sentimentos de aproximação dos terapeutas de ambos os sexos no atendimento inicial de pacientes mulheres vítimas de violência sexual. A partir disso, consideramos que terapeutas homens, assim como terapeutas mulheres, são capazes de empatizar e sintonizar-se afetivamente 
com as pacientes nesse momento do tratamento. Não foram observadas diferenças nas reações contratransferenciais em relação às pacientes vítimas de violência sexual em momentos distintos do desenvolvimento.

Foi identificado um predomínio de respostas de distanciamento nos terapeutas homens que atenderam pacientes vítimas de traumas não-sexuais, relacionados à violência urbana. Pensamos que esse achado pode dever-se ao sentimento de vulnerabilidade despertado nos terapeutas, em vista da maior e crescente prevalência de violência urbana na população masculina. O presente estudo forneceu diversas questões de pesquisa que podem contribuir para investigações futuras.

\section{Referências}

1. Freud S. As perspectivas futuras da terapêutica psicanalítica (1910). In: Edição standard brasileira das obras psicológicas completas de Sigmund Freud. Rio de Janeiro: Imago; 1970.

2. Heimann P. On countertransference. Int J Psychoanal. 1950;31:814.

3. Racker E. The meanings and use of countertransference. Psychoanal Q. 1957;26(3):303-57.

4. Winnicott D. Hate in the countertransference. Int J Psychoanal. 1949;30:69-75.

5. Barros EM. [Psychoanalysis and research]. Rev Bras Psiquiatr. 2006;28(3):169-70.

6. Eizirik CL. [Psychoanalysis and research]. Rev Bras Psiquiatr. 2006;28(3):171-2.

7. Fonagy P. An open door review of outcome studies in psychoanalysis. 2nd ed. London: International Psychoanalytical Association; 2002.

8. Gabbard GO. Countertransference: the emerging common ground. Int J Psychoanal. 1995;76(Pt 3):475-85.

9. Viederman M. The real person of the analyst and his role in the process of psychoanalytic cure. J Am Psychoanal Assoc. 1991;39(2):451-89.

10. Kernberg OF. The influence of the gender of patient and analyst in the psychoanalytic relationship. J Am Psychoanal Assoc. 2000;48(3):859-83.

11. Langs R, Rapp PE, Pinto A, Cramer G, Badalamenti A. Three quantitative studies of gender and identity in psychotherapy consultations. Am J Psychother. 1992;46(2):183-206.

12. Betan E, Heim AK, Zittel Conklin C, Westen D. Countertransference phenomena and personality pathology in clinical practice: an empirical investigation. Am J Psychiatry. 2005;162(5):890-8.

13. Eizirik M, Schestatsky S, Knijnik L, Terra L, Ceitlin LHF. Contratransferência e trauma psíquico. Rev Psiquiatr RS. 2006;28(3):314-20.

14. Pearlman LA, Saakvitne KW. The therapeutic relationship as the context for countertransference and vicarious traumatization. In: Pearlman LA, Saakvitne KW. Trauma and the therapist. New York: WW Norton; 1995. p. 15-34.
15. Dalenberg CJ. Countertransference and the treatment of trauma. Washington, DC: American Psychological; 2000.

16. Pires ACJ. Reação terapêutica negativa e impasse. In: Eizirik C, Schestatsky S, Aguiar R, eds. Psicoterapia de orientação analítica: fundamentos teóricos e clínicos. $2^{\mathrm{a}}$ ed. Porto Alegre: Artmed; 2005. p. 329-49.

17. Zavaschi ML, Graeff ME, Menegassi MT, Mardini V, Pires DW, Carvalho RH, et al. Adult mood disorders and childhood psychological trauma. Rev Bras Psiquiatr. 2006;28(3):184-90.

18. Kendler KS, Bulik CM, Silberg J, Hettema JM, Myers J, Prescott CA. Childhood sexual abuse and adult psychiatric and substance use disorders in women: an epidemiological and cotwin control analysis. Arch Gen Psychiatry. 2000;57(10):953-9.

19. Kessler RC, Davis CG, Kendler KS. Childhood adversity and adult psychiatric disorder in the US National Comorbidity Survey. Psychol Med. 1997;27(5):1101-19.

20. Mclntyre SM, Schwartz RC. Therapists, differential countertransference reactions toward clients with major depression or borderline personality disorder. J Clin Psychol. 1998;54(7):92331 .

21. Brody EM, Farber BA. The effects of therapist experience and patient diagnosis on countertransference. Psychoterapy. 1996;33(3):372-80.

22. Brasil, Ministério da Justiça. Disponível em: www.mj.gov.br/ senasp/estatisticas/mapacrime/ Mapacrime 2004_2005.pdf.

23. Leserman J. Sexual abuse history: prevalence, health effects, mediators, and psychological treatment. Psychosom Med. 2005;67(6):906-15.

24. Bardin L. Análise de conteúdo. Lisboa: Edições 70; 1977.

25. Moraes R. Análise de conteúdo. Educação. 1999;37:7-32.

26. Peleikis DE, Mykletun A, Dahl AA. The relative influence of childhood sexual abuse and other family background risk factors on adult adversities in female outpatients treated for anxiety disorders and depression. Child Abuse Negl. 2004;28(1):61-76.

27. Peleikis DE, Mykletun A, Dahl AA. Current mental health in women with childhood sexual abuse who had outpatient psychotherapy. Eur Psychiatry. 2005;20(3):260-7.

28. Holmqvist R, Armelius BA. The patients contribution to the therapist's countertransference feelings. J Nerv Ment Dis. 1996;184(11):660-6.

29. Faravelli C, Giugni A, Salvatori S, Ricca V. Psychopathology after rape. Am J Psychiatry. 2004;161(8):1483-5.

30. Gunther MS. Countertransference issues in staff caregivers who work to rehabilitate catastrophic-injury survivors. Am J Psychother. 1994;48(2):208-20.

31. Kinzie JD, Boehnlein JK. Psychotherapy of the victims of massive violence: countertransference and ethical issues. Am J Psychother. 1993;47(1):90-102.

32. Mogul KM. Overview: the sex of the therapist. Am J Psychiatry. 1982;139(1):1-11.

33. Eizirik CL. Masculinity, femininity and analytic relationship: countertransferential issues. In: Alcorta Garza A, ed. Psychoanalysis in Latin America. Monterrey: Fepal; 1995.

34. Zlotnick C, Elkin I, Shea MT. Does the gender of a patient or the gender of a therapist affect the treatment of patients with major depression? J Consult Clin Psychol. 1998;66(4):655-9.

35. Latts MG, Gelso CJ. Countertransference behavior and management with survivors of sexual assault. Psychoterapy 1995;32(3):405-15.

36. Goldfeld PRM, Wiethaeuper D, Bouchard MA, Terra L, Abuchaim C, Mardini V, et al. Mental states and countertransference in psychotherapists facing reports of traumatic events of mourning and sexual violence. Psychother Res. In press. 